\title{
Les praticiens à diplôme hors Union Européenne (PADHUE) en France : quand les hôpitaux ont recours à des médecins-migrants
}

\section{Victoire Cottereau}

\section{OpenEdition}

1 Journals

Édition électronique

URL : http://journals.openedition.org/rfst/433

DOI : $10.4000 /$ rfst.433

ISSN : 2492-3672

Éditeur

Espaces et SOciétés (UMR 6590)

Référence électronique

Victoire Cottereau, «Les praticiens à diplôme hors Union Européenne (PADHUE) en France : quand les hôpitaux ont recours à des médecins-migrants ", Revue francophone sur la santé et les territoires [En ligne], Miscellanées, mis en ligne le 13 mai 2015, consulté le 06 avril 2021. URL : http:// journals.openedition.org/rfst/433; DOI : https://doi.org/10.4000/rfst.433

Ce document a été généré automatiquement le 6 avril 2021.

La Revue francophone sur la santé et les territoires est mise à disposition selon les termes de la Licence Creative Commons Attribution - Pas d'Utilisation Commerciale - Partage dans les Mêmes Conditions 4.0 International. 


\title{
Les praticiens à diplôme hors Union Européenne (PADHUE) en France : quand les hôpitaux ont recours à des médecins-migrants
}

\author{
Victoire Cottereau
}

Depuis les années 1970, la démographie médicale est une préoccupation majeure en France. À cette époque, l'explosion du nombre d'étudiants dans les facultés de médecine fait craindre une future pléthore de médecins. Cette situation engendre alors la mise en place du numerus clausus et de plusieurs réformes de l'enseignement médical afin de limiter le nombre de praticiens formés. Depuis, des instituts de recherche (comme l'IRDES ${ }^{1}$ ) et des organismes d'État spécialisés (ONDPS ${ }^{2}$ ) publient de nombreux rapports et prévisions dans le but d'améliorer les connaissances relatives à la démographie des professions de santé. Pourtant, la démographie médicale française connaît actuellement une inégale distribution des médecins sur son territoire (Berlan, 2002 ; Descours, 2003). Celle-ci a pour conséquence de contraindre des populations habitant souvent des zones rurales ou montagneuses, à parcourir de longues distances pour pouvoir trouver, entre autres, des médecins généralistes libéraux (Chasles, Denoyel et Vincent, 2013). Ces profondes inégalités de densité médicale se doublent également de déséquilibres et de déficits parfois problématiques au sein des structures hospitalières publiques (Grimaldi, 2009). En effet, les nouveaux professionnels tendent à éviter d'exercer dans les hôpitaux de pôles urbains secondaires (Séchet et Vasilcu, 2012). Cette seconde conséquence, moins étudiée, nécessite un examen attentif afin de connaître et comprendre les phénomènes qui entravent le fonctionnement normal des établissements hospitaliers. Ainsi, en moins d'un demi-siècle, la démographie médicale française passe d'un état de trop-plein de médecins à une situation de pénurie partielle de praticiens diplômés en France. C'est dans ce contexte que l'État ouvre alors ses hôpitaux à des médecins venant de pays faisant partie de l'Union européenne puis à des praticiens venant de pays situés au-delà des frontières européennes. 
2 Cet article vise à apporter un éclairage sur une catégorie de médecins «à part ": les PADHUE (Praticiens à diplôme hors Union européenne). Les membres de cette catégorie ont comme seul dénominateur commun d'avoir obtenu leur diplôme de médecine dans un pays ne faisant pas partie de l'Union européenne (UE). La situation de ces médecins fait l'objet de peu de travaux précis, notamment parce que la législation à leur sujet est complexe et évolutive (voir figure $\mathrm{n}^{\circ} 1$ ). Ce constat nous porte à nous interroger sur la place et le rôle de ces migrants hautement qualifiés, ainsi que sur le système de santé français dans lequel ils évoluent. À la croisée du domaine des migrations internationales et du domaine de la géographie de la santé, cette étude analyse ce contexte spécifique au travers des parcours et projets migratoires des PADHUE en exercice dans les hôpitaux publics de la région Poitou-Charentes ${ }^{3}$.

3 Dans un premier temps, l'article reviendra sur les évolutions des cadres règlementaires qui ont conduit à l'arrivée de ces médecins en France. Dans un deuxième temps, l'article exposera les résultats d'une enquête menée auprès des structures hospitalières de la région Poitou-Charentes, afin de mieux comprendre les dynamiques régionales et locales des recrutements de PADHUE. Enfin, dans une troisième partie, nous ferons un focus sur les parcours et trajectoires de ces médecins. In fine, l'article discutera de la notion de carrière itinérante, à travers l'analyse des dynamiques migratoires et des mobilités de ces professionnels de la santé évoluant dans une situation inédite.

\section{Le métier de médecin en France : évolution d'une profession réglementée}

4 En France, la profession de médecin est soumise à un régime spécifique. L'article $\mathrm{L}$. 4111-1 du code la santé publique (CSP) pose trois conditions cumulatives pour pouvoir exercer la médecine :

- être titulaire d'un diplôme d'État de docteur en médecine délivré par la France ou par un État membre de l'Union européenne (une condition de diplôme),

- être de nationalité française, andorrane, être ressortissant d'un pays membre de l'UE ou être de nationalité tunisienne ou marocaine (une condition de nationalité),

- être autorisé à s'inscrire au Tableau de l'Ordre des médecins (une condition d'admission par la profession).

5 Analyser cette catégorie de médecin (PADHUE) implique de connaître et comprendre simultanément le contexte lié à la démographie médicale française et les dynamiques migratoires propres à ces travailleurs hautement qualifiés. Cette analyse étant rarement réalisée, nous proposons dans cette partie de revenir sur l'évolution des cadres législatifs qui a conduit aux trajectoires professionnelles de ces médecins.

\section{Entrouverture du métier aux médecins diplômés à l'étranger}

6 Longtemps réservée aux personnes de nationalité française et titulaires d'un diplôme français, la profession s'ouvre d'abord aux médecins ayant obtenu leur diplôme dans un pays membre de l'Union européenne. Dans le cadre de la construction européenne, la directive 75/362/CEE du Conseil des communautés européennes de 1975 leur permet de pouvoir librement pratiquer la médecine en France. Pourtant, les candidats européens sont peu nombreux à venir s'installer sur le territoire français et ils ne remplissent 
qu'une faible partie des postes vacants dans les hôpitaux. Face au faible impact de l'ouverture de la profession aux médecins diplômés en UE, l'État français décide dès la décennie 1980 d'attirer des praticiens à diplôme hors Union européenne (PADHUE ${ }^{4}$ ).

Figure 1 : Frise chronologique des principales législations à destination des PADHUE en France

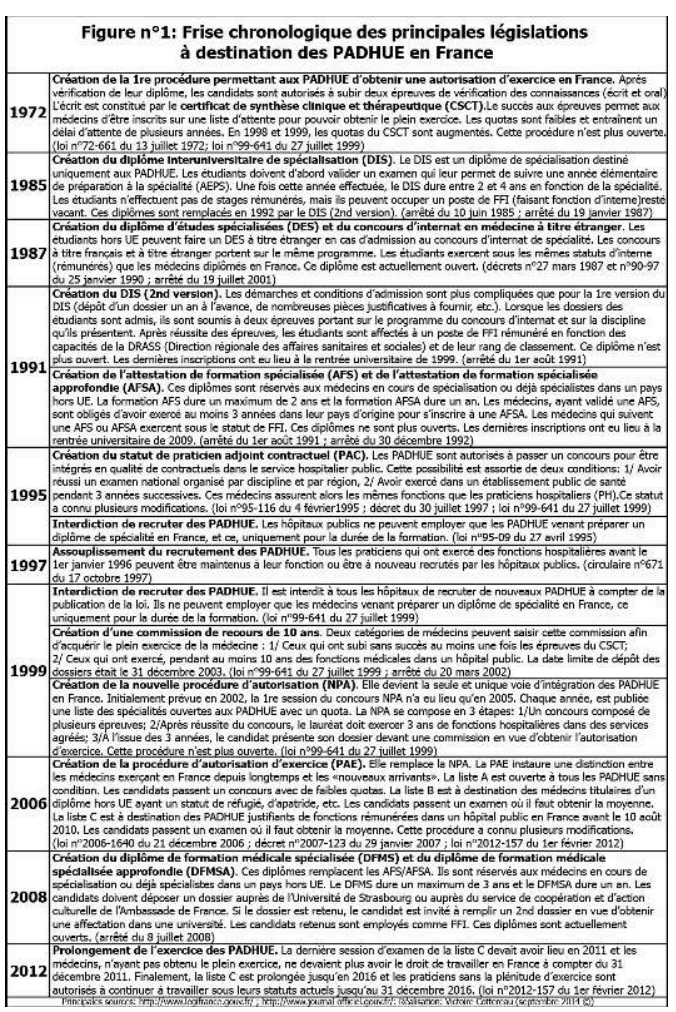

Sources : http://www.legifrance.gouv.fr ; http://www.journal-officiel.gouv.fr. Réalisation : V. Cottereau, 2014

7 En 1981 et $1987^{5}$, deux décrets accordent aux médecins étrangers ou titulaires d'un diplôme étranger le droit d'exercer dans les hôpitaux publics sous des conditions particulières (Hatzfeld, Boidé et Baumelou, 2009). L'État français crée également des cursus de spécialisation à destination des PADHUE dès 1985 (voir figure $\mathrm{n}^{\circ} 1^{6}{ }^{6}$ ). Pendant qu'ils préparent ces diplômes, les médecins sont autorisés à travailler en parallèle dans les hôpitaux publics. Cette autorisation de travail est soumise à un certain nombre de contraintes telles que l'interdiction d'exercer comme médecin libéral ou dans les établissements privés. Les PADHUE n'ont alors pas le droit de s'inscrire au Conseil national de l'Ordre des médecins (CNOM) et ils ont l'obligation de travailler sous des statuts peu rémunérateurs créés spécifiquement pour eux. À ce moment-là, cette législation hospitalière est probablement pensée par l'État comme une réponse ponctuelle ne devant pas perdurer.

8 Finalement, cette solution se perpétue, conduisant les professionnels concernés à se regrouper en syndicats et à médiatiser leur situation. En réponse, l'État crée des concours afin de permettre à ces médecins de pouvoir faire reconnaitre leurs diplômes et d'accéder aux mêmes statuts que leurs confrères diplômés en France et en UE. Chaque concours est envisagé comme étant le dernier dans son genre, devant permettre aux PADHUE d'intégrer pleinement le corps médical. Dans l'intention de 
mettre fin à ces migrations, chaque concours est également couplé d'une interdiction de recruter de nouveaux médecins à diplôme extraeuropéen.

9 Se trouvant toujours en contradiction avec des logiques de marché et de besoins, cette législation engendre des dérogations et des contournements mis en place par les structures hospitalières. Cela signifie qu'à chaque nouvelle interdiction d'effectuer des recrutements de PADHUE, les hôpitaux ont malgré tout continué à recruter, à la marge de la légalité. Cependant, si cela permet de maintenir des effectifs suffisants pour le bon fonctionnement de services hospitaliers, ces médecins se retrouvent sous contrat hors des législations en cours. Par conséquent, ils ne remplissent pas les conditions nécessaires pour passer des concours d'équivalence et doivent attendre la création d'une nouvelle législation en leur faveur. Ainsi, bien qu'indispensables dans les structures hospitalières, ces médecins sont soumis aux aléas d'une législation mouvante et évolutive (faite de périodes d'ouvertures et de fermetures visibles), mais également à des procédures de reconnaissance de diplômes particulièrement rigides et longues, avant d'être admis ou rejetés du corps médical français, ou encore maintenus en marge de celui-ci (voir figure $n^{\circ} 1$ ). Toutefois, depuis le durcissement de la législation à la fin des années 2000 et la mise en place de nouveaux diplômes plus réglementés à destination des PADHUE, les possibilités de contournements semblent se tarir. Cette dernière évolution de la législation annonce la fin de cette migration internationale. Désormais, le nombre de PADHUE en France devrait diminuer au fur et à mesure que ces praticiens partiront à la retraite.

\section{Les PADHUE : un objet de recherche}

10 En 2008, des membres de la Fédération des praticiens de santé (FPS) estiment le nombre de PADHUE en France à environ 17000 individus (Dalkilic, Meric et Trujillo Guiterrez, 2008). Ce nombre est approximatif, car les médecins qui ne possèdent pas la plénitude d'exercice ne sont pas autorisés à s'inscrire au CNOM. Aucun organisme d'État (Agence régionale de santé, Centre national de gestion, etc.) ne possède de données précises sur ces praticiens. Aujourd'hui, ces effectifs ne peuvent être connus que par l'intermédiaire des registres des ressources humaines des établissements hospitaliers qui les emploient. Qu'elle soit minimisée, amplifiée ou exacte, l'estimation du syndicat illustre de manière significative la force de travail et la place indispensable de ces praticiens dans le système de santé français.

11 Afin de recueillir des données sur ces médecins, notre méthodologie de recherche articule deux types d'approches: un pan quantitatif et un volet qualitatif. Le pan quantitatif, réalisé en 2011, a permis de collecter des informations sur les praticiens en exercice dans les hôpitaux de la région Poitou-Charentes. Un volet qualitatif, effectué entre 2012 et 2014, a consisté au recueil d'entretiens et de récits de vie biographiques (78 entretiens) auprès des médecins et du personnel administratif (direction / affaires médicales) des structures hospitalières.

Nous avons choisi d'utiliser l'enquête biographique auprès des praticiens parce que cette méthodologie est appropriée pour saisir la dynamique des processus en interaction dans un parcours biographique. Selon le groupe de réflexion sur l'approche biographique (1999), l'histoire de vie des personnes est un enchaînement continu d'évènements de nature diverse concernant, entre autres, leur famille, leur résidence et leur profession. Nous avons donc élaboré un outil permettant de recueillir le parcours 
biographique d'un individu en une seule rencontre, un minimum de temps et de façon à ce que les données biographiques recueillies soient les plus fiables possible. Inspirées de travaux comme ceux de Daniel Courgeau (1985), nous avons construit une enquête composée d'un entretien et d'une matrice biographique permettant de récolter simultanément les trajectoires dans trois domaines (familial, professionnel et migratoire). Ce type d'enquête s'est avéré efficace et nous a permis de vérifier l'hypothèse que c'est l'interaction de facteurs qui engendre un espace et un temps propre à chaque situation.

13 Cette actuelle recherche est donc fondée sur un croisement entre des données statistiques sur la démographie médicale en région Poitou-Charentes et des informations qualitatives sur le parcours de vie de médecins diplômés en dehors de l'Union européenne. Elle offre non seulement un nouveau regard sur les professionnels de santé et l'hôpital, mais elle apporte également des données inédites sur le système de santé français.

\section{Raisons et conséquences d'une démographie médicale déséquilibrée}

14 Selon l'atlas de la démographie médicale, au 1 er janvier 2013, avec un effectif total de 271970 médecins inscrits au tableau du CNOM, la France atteint un record historique de praticiens recensés sur son territoire (CNOM, 2013). Ce phénomène est principalement lié à une augmentation du nombre de médecins retraités au sein de cet effectif. Selon les projections effectuées en 2004 par l'ONDPS, l'âge moyen des médecins sera de 50,6 en 2015 contre 47,5 en 2008. Actuellement la France fait partie des pays de l'Organisation de coopération et de développement économique (OCDE) confrontés à la situation paradoxale de posséder un nombre satisfaisant de médecins tout en devant faire face à des phénomènes régionaux ou locaux de pénurie médicale partielle. Ce résultat est autant la conséquence de l'instauration du numerus clausus que d'évolutions sociétales.

\section{Le numerus clausus : un outil de régulation}

15 Le gouvernement français instaure le numerus clausus le 4 juin 1971. Avant cette date, pour passer en deuxième année de médecine, il suffisait d'obtenir un examen à l'issue de la première année d'études. À la fin des années 1960, le contexte économique français était plutôt prospère et les étudiants étaient de plus en plus nombreux à entrer à l'université. À cette époque, le gouvernement en place a alors limité le nombre d'étudiants en médecine. De surcroît, l'externat de médecine est supprimé en 1968. Par conséquent, à compter de cette date, tous les étudiants doivent exercer pendant trois ans des fonctions d'externe dans les hôpitaux dont les places sont limitées et non extensibles. C'est alors qu'est instauré un outil de régulation spécifique : le numerus clausus. La démographie médicale française devient donc gérée par des quotas d'étudiants, fixés annuellement par le gouvernement. À partir de là, dans un contexte où se met en place une " profession protégée » (Lochard, Meilland et Viprey, 2007, p. 88) effrayée par le spectre de la pléthore médicale, les différents gouvernements ne cessent de baisser les quotas du numerus clausus jusqu'à début des années 2000. Pendant près de 30 ans, le pays forme de moins en moins de médecins. Cette situation a comme 
conséquence immédiate de créer un manque d'internes dans les structures hospitalières et comme conséquence à long terme de " priver la France d'aujourd'hui d'environ 50000 médecins » (Wallach, 2011, p. VII). Ainsi, dès le début des années 1980, les établissements hospitaliers voient leur nombre d'internes s'effondrer et doivent trouver une solution pour continuer à fonctionner normalement sans entacher leur budget. En raison du faible nombre de candidats européens, la réponse est trouvée par le biais d'une «migration de blouses blanches » venant de pays ne faisant pas partie de l'Union européenne.

\section{Les facteurs de déséquilibre de la démographie médicale}

16 Malgré l'arrivée de nombreux PADHUE dès la décennie 1980, d'autres facteurs non négligeables continuent de bouleverser la démographie médicale française. Tout d'abord, la mise en place de l'internat de spécialité, en 1984, instaure une seule et unique voix de spécialisation. Celle-ci a pour conséquence d'instaurer une seconde sélection au sein des études médicales avec comme impact direct de limiter le nombre de spécialistes. Puis, au début des années 1990, période où la crainte d'un sureffectif de médecins est à son maximum, plusieurs mesures visant à diminuer le nombre de praticiens sont mises en place (Dormont et Samson, 2008), notamment des incitations de reconversion et de départs à la retraite. Dans le même temps, le vieillissement de la population française entraîne une demande accrue auprès du système de santé et oblige celui-ci à s'adapter en recrutant de nouveaux médecins et en créant notamment de nouveaux services de gérontologie et de gériatrie (Bliez, 2006). D’autres éléments de contexte interviennent également, notamment la féminisation de la profession médicale. Dans les années 1990, environ 30\% des médecins étaient des femmes contre plus de $40 \%$ dans les années 2000 (Lapeyre et Le Feuvre, 2005). Selon l'ONDPS (2004), elles représenteraient $52 \%$ des médecins en activité en 2025. Or, cette féminisation nécessiterait de former davantage de médecins, car les femmes sont plus fréquemment demandeuses d'emplois à temps partiel que leurs collègues masculins. On observe également un changement de représentation du métier par les jeunes praticiens (Lévy, 2011). Actuellement, de nombreux médecins, hommes et femmes, souhaitent pouvoir concilier leur vie professionnelle et leur vie personnelle. Cette évolution occasionne une défection pour les zones les plus isolées, afin que le conjoint du praticien puisse trouver un emploi. Elle engendre également des déficits très marqués dans certaines spécialités qui laissent peu de place à la vie de famille, comme la psychiatrie ou la médecine générale (Bessière, Breuil-Genier et Darrine, 2004). Enfin, la réduction du temps de travail ${ }^{7}$ a des impacts sur la disponibilité des professionnels de santé, car elle conduit notamment à intégrer les périodes de permanence sur place (les gardes) dans le temps effectif de travail (Tonneau, 2003).

17 Ces multiples facteurs sont à l'origine d'un déséquilibre de la démographie médicale et de nombreux bouleversement dans un secteur hospitalier qui s'institutionnalise à la fin des années $1950^{8}$. Ces évolutions ont amplifié le manque de médecins dans les hôpitaux généraux et ont accru l'inégale répartition des médecins. Elles ont également eu pour conséquence de pérenniser la place des PADHUE dans le système de santé français. 


\section{État des lieux des PADHUE en région Poitou-Charentes}

18 Le recensement effectué auprès de l'intégralité des structures hospitalières publiques de la région Poitou-Charentes (soit 30 établissements) dénombre 2088 praticiens en exercice au $1^{\mathrm{er}}$ janvier $2011^{9}$. Parmi ces praticiens, 59,6\% sont des hommes et 40,4\% sont des femmes. Au sein de l'ensemble de ces médecins, 91\% sont diplômés dans un pays membre de l'Union européenne (1901 individus) et $9 \%$ sont diplômés hors Union européenne (187 individus). Il est important de noter que le nombre de PADHUE est probablement un peu plus élevé dans la réalité. Certains hôpitaux ont parfois exclu de cette catégorie des praticiens dont les pays font aujourd'hui partie de l'Union européenne, mais qui sont arrivés en France avant que leur pays n'ait adhéré à l'UE ${ }^{10}$. Des responsables administratifs reconnaissent également avoir pu classer des PADHUE dans la catégorie "médecins diplômés en France » en raison de leur ancienneté et du fait qu'ils pensent qu'ils ont fait leur cursus universitaire en France.

19 La carte $\mathrm{n}^{\circ} 1$ représente la localisation de l'ensemble des praticiens en exercice dans les hôpitaux publics de la région tout en distinguant les différents lieux d'obtention du doctorat de médecine (France, UE et hors UE). Cette représentation cartographique met en relief deux points essentiels. Premièrement, on constate que près d'un médecin sur dix (9\%) a obtenu son diplôme de médecine hors UE. Deuxièmement, on observe que des PADHUE sont employés dans la quasi-totalité des établissements, à l'exception de quelques petites structures (ex-hôpitaux locaux). En outre, leur répartition est très hétérogène entre les départements et les structures hospitalières. En effet, les hôpitaux situés dans le département des Deux-Sèvres emploient 57 PADHUE contre seulement 24 pour les établissements situés en Vienne. Pourtant, en raison de la présence du CHU, ce département possède un effectif total de médecins (663 individus) beaucoup plus élevé que le département frontalier (401 individus). On remarque également des pourcentages de PADHUE très faibles au sein de structures $(0,4 \%$ des praticiens du $\mathrm{CH}$ de La Rochelle) et des pourcentages importants au sein d'hôpitaux situés dans le même département (38,3\% des médecins du CH de Saint-Jean-d'Angély). Ces observations soulignent le particularisme de chaque établissement. De multiples éléments sont à prendre en compte pour comprendre et analyser la place des PADHUE au sein des équipes hospitalières (les différentes politiques de recrutement des hôpitaux, les choix effectués par les PADHUE, la localisation des structures vis-à-vis du CHU ou d'autres grandes structures très attractives pour les praticiens, la localisation géographique des établissements, la taille et l'équipement des plateaux techniques, la réputation des structures et des services, etc.).

20 La carte $n^{\circ} 1$ localise les PADHUE sans distinguer ceux qui possèdent le plein exercice de la médecine, d'ordinaire très recherchés par les hôpitaux, de ceux qui ne la possèdent pas, souvent recrutés à défaut d'autres praticiens (Drexler, 2008). Son objectif principal est de présenter la répartition des médecins sur le territoire tout en pointant du doigt la place indispensable des PADHUE dans le fonctionnement des structures hospitalières.

21 L'analyse des services d'affectation des médecins à diplôme extraeuropéen en région Poitou-Charentes apporte des informations beaucoup plus fines sur le rôle de ces médecins à l'intérieur des établissements. Les données montrent que $72,7 \%$ des PADHUE travaillent dans seulement huit services: $26,7 \%$ aux urgences, $12,8 \%$ en cardiologie, $7 \%$ en chirurgie, $7 \%$ en médecine générale ou polyvalente, $5,3 \%$ en 
radiologie-imagerie médicale, $4,8 \%$ en gynécologie obstétrique, $4,8 \%$ en psychiatrie et $4,3 \%$ en anesthésie-réanimation. La mise en lien de ces chiffres avec les discours des PADHUE sur leurs expériences professionnelles (voir plus loin) montre que ces médecins jouent visiblement un rôle d'ajustement dans le système hospitalier français. Effectivement, ces praticiens exercent le plus souvent dans des services difficiles et/ou les moins prisés par leurs confrères diplômés en France.

Carte 1 : Localisation des praticiens en exercice dans les hôpitaux publics de la région PoitouCharentes au 1er janvier 2011

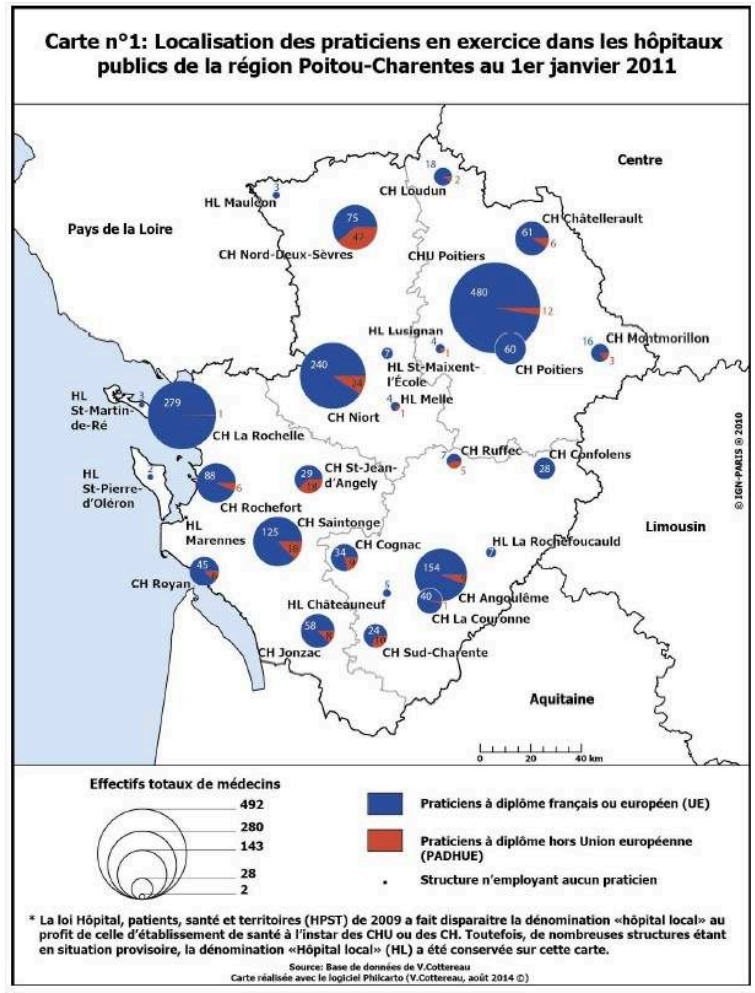

Source : Base de données V. Cottereau. Réalisation : V. Cottereau, 2014

\section{Parcours et trajectoires des PADHUE : des carrières itinérantes}

L'analyse des données quantitatives et qualitatives nous a permis d'extraire de nombreux résultats sur les praticiens et sur le système dans lequel ils évoluent. Elle nous a également servi à étudier les impacts de ce système sur les carrières des médecins, et inversement. Dans le cadre de cette dernière partie, nous allons nous pencher sur les projets migratoires des médecins ainsi que sur les raisons de leur installation en France. Cette analyse sera illustrée par quelques citations extraites d'entretiens que nous avons réalisés auprès de PADHUE. Enfin, tout en nous appuyant sur des cartes représentant la mobilité professionnelle et résidentielle de deux médecins, nous nous arrêterons sur la construction des carrières des PADHUE en France. 


\section{Les projets migratoires des PADHUE}

Les PADHUE qui travaillent dans les hôpitaux publics de la région étudiée ont obtenu leur doctorat de médecine ${ }^{11}$ dans vingt-six pays différents. Sur le tableau ${ }^{\circ}{ }^{0} 1$ sont représentées les grandes parties du monde dans lesquelles les PADHUE ont validé leur titre de docteur. Ces médecins ont majoritairement obtenu leur doctorat dans deux pays du Maghreb : 56 praticiens sont diplômés en Algérie (29,9\% des PADHUE recensés) et 43 ont obtenu leur diplôme au Maroc (23\%). À la suite de ces deux pays arrivent la Syrie (11,2\%), Madagascar $(9,6 \%)$, le Liban $(2,7 \%)$, la Tunisie $(2,7 \%)$, la Russie $(2,1 \%)$ et l'Ukraine (2,1\%). Tous les autres pays (18) ont une part individuelle inférieure à $2 \%$.

Tableau 1 : Effectifs et pourcentages par lieux d'obtention du doctorat des PADHUE en exercice dans les hôpitaux publics de la région Poitou-Charentes au 1 er janvier 2011

\begin{tabular}{|c|c|c|}
\hline $\begin{array}{c}\text { Lieux } \\
\text { d'obtention du } \\
\text { doctorat }\end{array}$ & Effectifs & Pourcentages \\
\hline $\begin{array}{c}\text { Afrique du Nord } \\
\text { (Maghreb + } \\
\text { Éqypte) }\end{array}$ & 107 & $57,2 \%$ \\
\hline Afrique Noire & 33 & $17,6 \%$ \\
\hline Moyen-Orient & 30 & $16,0 \%$ \\
\hline $\begin{array}{c}\text { Asie } \\
\begin{array}{c}\text { Europe (hors } \\
\text { pays EU et } \\
\text { incluant la } \\
\text { Russie) }\end{array}\end{array}$ & 4 & $2,1 \%$ \\
\hline Amérique du Sud & 3 & $1,6 \%$ \\
\hline $\begin{array}{c}\text { Donnée } \\
\text { manquante }\end{array}$ & 1 & $0,5 \%$ \\
\hline TOTAL & $\mathbf{1 8 7}$ & $\mathbf{1 0 0 , 0} \%$ \\
\hline
\end{tabular}

Source : Enquêtes personnelles / Réalisation : Victoire Cottereau

L'étude des pays d'obtention du doctorat de médecine des PADHUE dévoile que ces médecins sont majoritairement issus d'anciennes colonies et de protectorats français. Il est important de souligner cette information, car elle a souvent été décisive lors de l'élaboration du projet initial des praticiens. En effet, la plupart d'entre eux déclarent avoir choisi de venir en France en raison de la langue, des liens historiques forts entre leurs pays d'origine et la France ainsi que pour la renommée du système de santé français.

«Quand je suis venu, je n'avais pas l'idée de rester, je voulais profiter du savoirfaire français, de la renommée de certains professeurs français. » (Youssef, homme, 53 ans, né et diplômé au Maroc, arrivé en France en 1987)

À tous ces facteurs, il faut ajouter la possibilité d'effectuer en France une spécialité à partir de 1985 (voir figure $n^{\circ}$ 1). La volonté de se spécialiser est la principale raison de départ évoquée par les médecins. Ce constat illustre que les diplômes de spécialisation mis en place par la France à destination des médecins diplômés à l'étranger attirent énormément de PADHUE. Mais ce désir de se spécialiser en France est souvent lié à d'autres raisons, de la simple envie d'aller finir ses études dans un pays étranger, à l'absence de moyens financiers ${ }^{12}$ ou d'écoles de spécialisation dans leur pays d'origine 
${ }^{13}$ en passant par la rencontre d'une personne vivant en France ou la volonté de venir faire hospitaliser un proche. Parfois, cette volonté s'associe à des raisons politiques ou à des conflits au sein du pays de départ. Toutefois, une faible partie des praticiens déclarent avoir quitté leur pays uniquement pour fuir la guerre ou des situations inacceptables à leurs yeux.

«Moi, mon projet était clair. Je suis parti dans des circonstances assez difficiles parce qu'il y avait la montée de l'intégrisme en Algérie, il y avait l'instabilité, entre guillemets la guerre civile, c'était 1991. [...] Dans ma tête je partais pour 4 ou 5 ans et je revenais en Algérie.» (Farid, homme, 44 ans, né et diplômé en Algérie, arrivé en France en 1991)

En définitive, les motivations qui ont engendré le départ en France sont très différentes en fonction du pays de naissance, du moment de l'arrivée en France et de raisons personnelles. Toutefois, il ressort clairement de l'analyse des projets initiaux des médecins à diplôme extraeuropéen que cette migration ne s'est que minoritairement construite par l'intermédiaire de facteurs économiques.

\section{Les raisons de l'installation en France}

La majeure partie des médecins expliquent avoir eu comme projet initial de venir en France de façon temporaire, le plus souvent pour devenir spécialiste et avoir une expérience dans le système de santé français. D'ailleurs, la plupart d'entre eux indiquent avoir été déçus par les formations de spécialité, par la non-reconnaissance de leur diplôme et/ou par les conditions de travail en France. Nous avons pu remarquer que les médecins ont souvent une conception utopiste de la vie en France et qu'ils manquent d'informations et de renseignements sur le système de santé lors de l'élaboration de leur projet migratoire. Ce n'est qu'une fois arrivés sur place que de nombreux médecins ont découvert que leur diplôme de médecine n'est pas reconnu en France. Ils ont alors également appris qu'ils doivent exercer sous des statuts précaires en attendant d'obtenir le plein exercice de la médecine.

\footnotetext{
"Pour vous dire quand je suis arrivé, je suis allé au Conseil de l'Ordre et ils étaient surpris parce que je leur ai dit que je venais faire une demande pour que je puisse exercer en fait. Ils m'ont dit «vous venez d'où? ». Je leur ai dit que je venais du Maroc, que j'ai un diplôme marocain et ils ont dit "Oulala, doucement, doucement ». C'est là que j'ai compris que c'est très compliqué. » (Abbad, homme, 47 ans, né et diplômé au Maroc, arrivé en France en 1992)
}

Malgré un projet d'installation de courte durée en France et un certain désenchantement vis-à-vis du système de santé français, cette simple mobilité estudiantine se transforme fréquemment en migration internationale. Les raisons qui poussent ces praticiens à rester sont multiples et variées. Les causes les plus évoquées par les médecins sont d'ordre familial telles qu'un mariage, une naissance ou les problèmes de santé d'un membre de leur famille.

« On rencontre la femme de sa vie et puis voilà on reste, c'était ça. » (Nacer, homme,

57 ans, né et diplômé au Maroc, arrivé en France en 1982)

29 Les difficultés de réadaptation liées à une dégradation économique du pays de naissance ou une évolution des mentalités et de la manière de vivre sont également des facteurs omniprésents dans les propos de nombreux PADHUE. Certains médecins nous ont notamment expliqué être retournés s'installer dans leur pays d'origine. Ils sont finalement revenus, car leurs enfants ne s'y plaisaient pas, ou parce qu'eux-mêmes 
n'arrivaient à se réadapter au fonctionnement de leur ancien système de santé. Pour d'autres, les projets de retour dans le pays d'origine sont parfois ajournés ou repoussés en raison de conflits armés ${ }^{14}$ ou d'instabilités politiques.

\begin{abstract}
«Avant la chute du régime, je ne voulais pas rester ici. L'objectif était de faire une formation dans une spécialité et retourner au pays. Six mois après le régime est parti et il y a eu la troisième guerre du Golfe en 2003. Là j'ai changé mon projet et je commence à réfléchir à rester en France ou partir ailleurs comme en Angleterre, parce que nous c'était l'Angleterre l'objectif, pas la France. » (Issam, homme, 37 ans, né et diplômé en Irak, arrivé en France en 2002)
\end{abstract}

30 Les praticiens sont ainsi amenés à rester ou à revenir en France pour des raisons d'ordre sociétal, familial, professionnel, ou encore personnel. Ce caractère multifactoriel a pour conséquence d'engendrer chez certains médecins un report permanent du projet de retour et une difficile acceptation que celui-ci n'aura probablement jamais lieu. Enfin, nous avons constaté que les deux éléments qui mettent le plus souvent un point final au projet de rentrer sont l'obtention de l'autorisation d'exercice ${ }^{15}$ et de la nationalité française. De nombreux médecins reconnaissent que l'acquisition de la reconnaissance, de la sécurité de l'emploi et d'une meilleure rémunération a stoppé leur projet de repartir. Aujourd'hui, la plupart des PADHUE déclarent être fiers de leur parcours professionnel et de ce qu'ils accomplissent en France.

«Oui, je suis fier de moi. Honnêtement je suis fier de moi parce que malgré tous les obstacles je suis arrivé à m'en sortir. Bon moi je dis toujours le travail ça paye un jour ou un autre donc il n'y a pas de problème.» (Assim, homme, 52 ans, né et diplômé au Maroc, arrivé en France en 1988)

31 Les projets de ces migrants hautement qualifiés associés à une législation spécifique à leur égard, elle-même née d'un besoin de répondre rapidement à un manque de médecin dans le système de santé français, suscitent des parcours professionnels particuliers et uniques.

\title{
Des carrières itinérantes
}

32 Contrairement aux médecins diplômés en France qui ont une faible mobilité durant leur parcours professionnel (Picheral et Vigneron, 1993), les PADHUE changent souvent de villes et d'établissements hospitaliers en raison de la législation particulière à laquelle ils sont soumis. Celle-ci entraîne une certaine instabilité et précarité - voire même un déclassement par rapport au pays d'origine-durant la période où leur diplôme de médecine n'est pas reconnu. Durant les années où ils ne possèdent pas la plénitude d'exercice, les médecins sont souvent contraints d'effectuer des bifurcations par rapport à leur projet initial, d'élaborer des stratégies, de saisir des opportunités, ou encore de faire appel à leurs connaissances (réseaux personnels et professionnels) pour trouver du travail. Afin de pouvoir saisir ces parcours dans toute leur complexité, nous avons choisi de les étudier à travers la notion de carrière itinérante .

33 Le concept de carrière, élaborée par le sociologue Howard Becker (1985), permet de dépasser la conception classique de la carrière professionnelle, décrite comme une succession de postes ou d'emplois occupés. Par conséquent, nous avons décidé de le redéfinir de façon plus étroite et d'en faire découler la notion de carrière itinérante . Celle-ci permet un recentrement sur l'étude des carrières professionnelles s'inscrivant en dehors du cadre commun. La carrière itinérante est définie ici comme une succession 
d'étapes, d'emplois et de statuts professionnels s'effectuant dans une grande mobilité géographique et/ou avec des changements de positions disciplinaires, hiérarchiques (ascendants ou descendants) ou sectoriels (secteurs public et privé) au sein d'un domaine d'activités. L'objectif de la carrière itinérante est d'arriver à se maintenir dans ce même domaine dans l'attente d'une éventuelle reconnaissance des compétences et d'une intégration complète au sein du corps de métier. Toutes ces étapes se réalisent dans une grande incertitude. Les praticiens n'ont aucune assurance qu'ils pourront intégrer pleinement le corps médical français.

34 Dans le cadre de cette étude, les PADHUE peuvent rencontrer deux formes de carrières itinérantes différentes (celles-ci peuvent être cumulables) :

- La carrière itinérante au sens spatial : le médecin est très mobile au sein du territoire français.

Il va partout où il trouve des postes. (voir carte $n^{\circ} 2$ )

- La carrière itinérante au sens hiérarchique, disciplinaire ou sectoriel : le médecin est mobile au sein même du corps médical. Il peut changer de spécialité médicale, exercer une profession dans le secteur médical / paramédical ou travailler dans le secteur privé. (voir carte $\left.n^{\circ} 3\right)$

La carrière itinérante prend différentes formes en fonction de plusieurs facteurs : le moment de l'arrivée en France, la ville d'arrivée, les contacts et connaissances, la spécialité, les centres hospitaliers dans lesquels les praticiens exercent au fil de leur parcours, les moyens financiers, les objectifs, les attentes et la personnalité de l'individu, la situation familiale.

\section{La carrière itinérante au sens spatial}

36 La législation à l'attention des médecins diplômés hors UE est mouvante et faite de cycles d'ouvertures et de fermetures. Pendant une quarantaine d'années se succèdent plusieurs périodes où les structures hospitalières n'ont normalement pas le droit d'employer de praticiens diplômés hors UE. Les PADHUE doivent alors faire preuve d'adaptation entre les moments où il est facile de trouver un emploi de médecin et les périodes où il est quasiment impossible de travailler comme praticien. Quelques rares médecins n'ont pas besoin d'élaborer des stratégies, car ils arrivent en France durant une période propice à l'embauche de PADHUE ou parce qu'ils ont les moyens de ne pas travailler lorsqu'ils préparent une procédure d'autorisation d'exercice. Mais, la plupart des médecins doivent adopter différentes stratégies pour pouvoir continuer à travailler. Certains d'entre eux font le choix d'être mobiles spatialement (voir carte $\mathrm{n}{ }^{\circ}$ 2). Les mobilités sont différentes d'un individu à l'autre, car de multiples facteurs influencent chaque parcours.

- Suivre l'offre et la demande. Jusqu'au début de la décennie 2010, la réglementation concernant les PADHUE venant en France pour obtenir des diplômes complémentaires les autorise à occuper des postes d'internes sous le statut de faisant fonction d'interne (FFI). Les changements d'internes dans les hôpitaux français ont lieu tous les six mois. Lors des attributions de postes, une priorité absolue est accordée aux internes français, ce qui oblige les PADHUE à se déplacer pour prendre les postes laissés vacants.

«On se débrouillait pour trouver les stages, on pouvait les trouver dans d'autres départements alors du coup ça nous obligeait à parcourir de longues distances. Parce qu'en fait on donnait les postes d'abord aux internes français et s'ils n'avaient pas choisi les postes alors là on pouvait aller chercher chez les étrangers. Donc on 
faisait tout ce parcours un peu bizarroïde.» (Jules, homme, 60 ans, né en Centrafrique et diplômé en URSS ${ }^{16}$, arrivé en France en 1983)

- Être mobile pour pouvoir contourner les législations . Les PADHUE n'ont parfois pas ou plus le droit d'exercer en fonction de la période à laquelle ils arrivent ou des diplômes qu'ils effectuent. Jusqu'au durcissement de la législation en 2010, beaucoup d'entre eux optent pour la solution qui consiste à postuler dans de petits hôpitaux soumis à de sérieux problèmes de recrutement. Dans d'autres cas, il suffit aux médecins de changer d'hôpital pour contourner une loi qui leur interdit d'exercer à l'issue de l'obtention d'un diplôme ou parfois pour négocier un meilleur statut.

«Le chef ne peut pas me renouveler (plus de deux fois parce qu'il faisait une attestation de formation spécialisée approfondie) même s'il a envie de me garder, il ne peut pas me renouveler. Par contre quand je change d'hôpital, la loi disparait. Mais c'est quoi cette loi ?» (Régis, homme, 43 ans, né et diplômé au Bénin, arrivé en France en 2002)

Carte 2 : Parcours professionnel et résidentiel d'un PADHUE de son arrivée en France en 2004 à 2013. Nahid, homme, 37 ans (1976), né en France et diplômé en Syrie

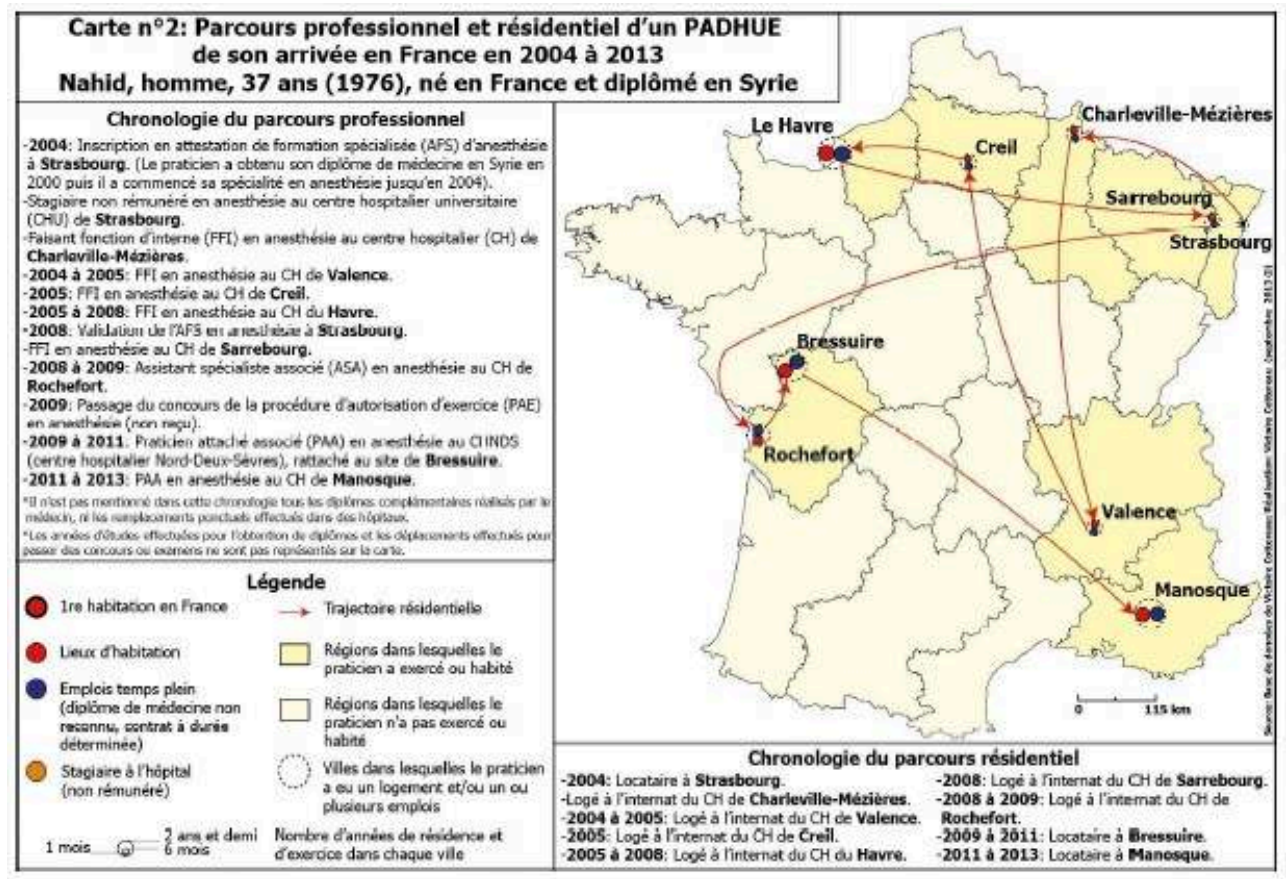


Carte 3 : Parcours professionnel et résidentiel d'un PADHUE de son arrivée en France en 2004 à 2013. Sariaka, femme, 39 ans (1974), née et diplômée en Madagascar

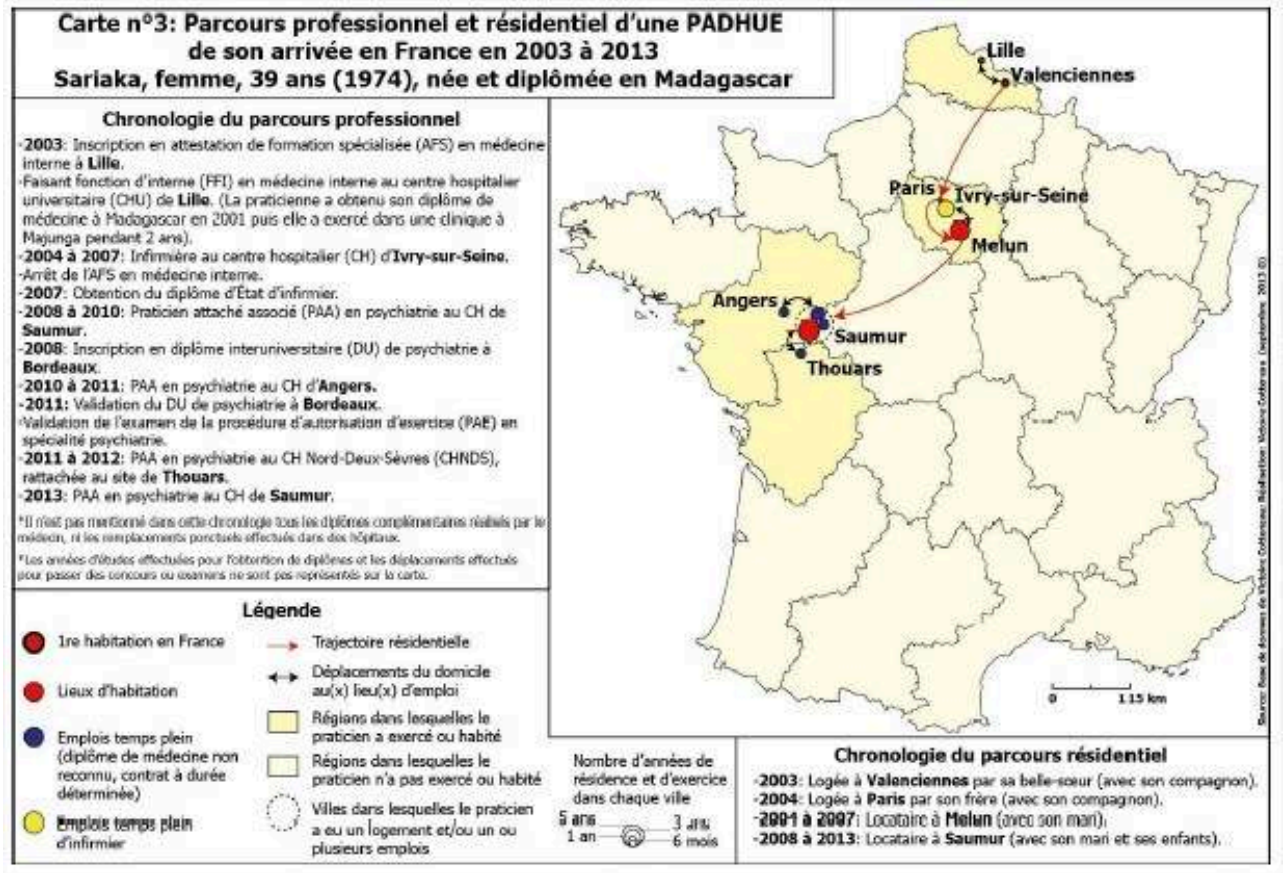

- Quitter la région parisienne. Selon les médecins, la région parisienne est le territoire où il est le plus difficile de trouver un poste de façon durable en France. Une bonne partie d'entre eux n'y ont que difficilement obtenu des postes. Lorsqu'ils y sont parvenus, c'était généralement pour une période de six mois ou pour un poste de nuit, voire même non rémunéré. Cette situation contraint des praticiens à partir en province pour avoir une situation plus stable.

«Il y avait des postes ici (dans un hôpital situé en Charente), il y avait une opportunité et il y avait des collègues que je connais déjà qui voulaient que je vienne, mais je ne voulais pas quitter Paris. [...] À Paris et dans la proche banlieue c'est pareil, on a un poste bouche-trou à faire la paperasse et on ne progresse pas quoi, pour avoir un poste de PH il faut se bagarrer. » (Asad, homme, 48 ans, né et diplômé en Algérie, arrivé en France en 1991)

- Partir parce que le contrat n'est pas renouvelé, pour être respecté ou pour être mieux rémunéré . Les contrats des PADHUE sont de courtes durées lorsqu'ils n'ont pas la plénitude d'exercice (généralement six mois). Lorsqu'ils ne sont pas renouvelés, ils n'ont pas d'autre choix de postuler dans d'autres structures s'ils veulent continuer à exercer leur profession. D'autres disent qu'il est nécessaire de changer régulièrement d'établissement s'ils veulent être respectés par leurs confrères diplômés en France et ne pas être consignés aux tâches ingrates. Plus occasionnellement, certains indiquent avoir quitté un hôpital parce qu'un autre leur a proposé un échelon de rémunération plus élevé.

« En fait pourquoi j'ai fait 36 trucs en même temps? Parce que j'ai toujours refusé d'être dépendant de quelqu'un ou l'esclave de quelqu'un donc là j'étais libre. [...] C'est pour ça que j'ai été dans plusieurs endroits, comme ça je dépendais de personne, je pouvais quitter dès qu'on m'embêtait. » (Larbi, homme, 49 ans, né et diplômé au Maroc, arrivé en France en 1984)

37 L'analyse des parcours des PADHUE indique que les médecins sont mobiles et changent régulièrement de structures afin de pouvoir exercer leur profession en France sans encore posséder la plénitude d'exercice. Les médecins qui ont une carrière itinérante au 
sens spatial sont souvent des praticiens qui souhaitent privilégier la pratique de leur métier à l'installation dans un lieu désiré.

\section{La carrière itinérante au sens hiérarchique, disciplinaire et sectoriel}

38 Durant leur parcours en France, près de la moitié des PADHUE enquêtés ont occupé d'autres emplois et/ou ont changé de spécialité médicale (voir carte $\mathrm{n}^{\circ} 3$ ). Ces choix peuvent être volontaires, stratégiques, nécessaires ou obligatoires.

- La relégation à un statut médical inférieur . De nombreux praticiens exercent un autre métier durant leur parcours en France. Le plus souvent, il s'agit de professions du secteur médical (infirmier, aide-soignant, etc.). Une partie des PADHUE font cela en attendant de trouver un poste de médecin ou pour compléter leurs revenus. En revanche, d'autres exercent un autre métier pendant plusieurs années, car ils ne trouvent pas de poste de médecin ou parce qu'ils ne savent pas qu'ils peuvent en obtenir un.

«Parce qu'avec mon poste de FFI (faisant fonction d'interne) je gagnais des clopinettes. À l'époque (en 1997) ce n'était même pas 4000 francs (environ 600 euros) et avec mon poste d'infirmier je gagnais 15000 francs (environ 2300 euros).» (Idris, homme, 53 ans, né et diplômé en Algérie, arrivé en France en 1990)

- L'abandon d'une spécialité et/ou l'acquisition d'une nouvelle spécialité . Les PADHUE savent que certaines disciplines, comme les urgences ou la psychiatrie, offrent de nombreux postes et des perspectives d'emploi à long terme. Le changement de discipline est souvent une stratégie dans l'objectif de trouver plus facilement un emploi durable avec une perspective de carrière ascendante. Tandis que des médecins généralistes choisissent de se spécialiser en raison du nombre de places limitées dans les services de médecine générale ou polyvalente, des spécialistes décident d'abandonner leurs spécialités lorsqu'ils n'arrivent pas à trouver de postes dans leur domaine de prédilection.

« Début 2007 j'ai commencé à me poser des questions : combien d'années je vais passer à chercher en médecine nucléaire ? Donc, en janvier 2007, j'ai commencé à penser à faire de la psychiatrie, les urgences ou la gériatrie [...] parce que ce sont les trois spécialités qui recrutent le plus, point. Moi je ne me voyais pas faire les urgences, je ne me voyais pas perdre ma tête en psychiatrie et par défaut gériatrie.» (Mahandry, homme, 41 ans, né et diplômé à Madagascar, arrivé en France en 1997)

- Accepter un travail illégal. Bien que ce soit illicite, des PADHUE ont expliqué avoir exercé dans le secteur privé durant la période où ils ne possédaient pas le plein exercice de la médecine. La plupart d'entre eux choisissent cette solution de façon temporaire le temps de trouver un autre emploi.

«J'ai travaillé dans des cliniques, mais ça c'était sans être déclaré. Je faisais aidechirurgien, je donnais un petit peu des coups de main, mais sans plus. » (Abbad, homme, 47 ans, né et diplômé au Maroc, arrivé en France en 1992)

La législation française à l'égard des PADHUE engendre un nouveau profil de carrière, celui de carrière itinérante. Celle-ci met des individus très qualifiés et indispensables au système de santé français face à des parcours très complexes. La carrière itinérante ne s'arrête pour s'orienter vers une carrière "classique " qu'à partir du moment où les médecins ont la reconnaissance de leur diplôme et leur inscription au CNOM. Une carrière "classique» de médecin diplômé en France signifie que les praticiens effectuent très peu de mouvements migratoires (Picheral et Vigneron, op.cit.) et qu'ils ont la liberté d'exercer où ils le souhaitent. Les carrières itinérantes se déclinent différemment en fonction des caractéristiques de chaque individu. Si certains ont une 
carrière itinérante réduite, d'autres sont contraints de combiner plusieurs types de carrières itinérantes au fil de leur parcours pour rester dans le milieu hospitalier.

\section{Conclusion}

40 Cette recherche mêlant collectes de données quantitatives et qualitatives a permis de dresser un portrait précis des PADHUE et d'établir un état des lieux inédit à l'échelle de la région Poitou-Charentes. Elle souligne le rôle d'ajustement et la place essentielle de ces médecins-migrants dans les hôpitaux publics. L'analyse des projets migratoires de ces médecins montre que les motivations économiques n'interviennent que faiblement dans cette migration. Initialement, la majorité des PADHUE sont motivés par les diplômes de spécialisation proposés par la France aux praticiens à diplôme extraeuropéen. Les facteurs et évènements personnels, familiaux ou encore sociétaux entraînent souvent un abandon du projet initial, qui est de rentrer dans leurs pays d'origine. La mobilité estudiantine se métamorphose graduellement en migration internationale. L'analyse des discours et des parcours de ces médecins révèle des carrières marquées par la complexité et les stratégies d'adaptation. La notion de carrière itinérante permet de saisir toutes les étapes par lesquelles passent les PADHUE dans l'objectif d'intégrer complètement le corps de métier. De la grande mobilité spatiale au changement temporaire de fonction, ces médecins font preuve d'une forme constante d'adaptation forgée d'opportunités, de stratégies ou encore de coups de hasard. Finalement, l'étude des trajectoires et des mobilités des PADHUE nous renseigne autant sur les dynamiques spatiales et temporelles liées à l'organisation du système de santé hospitalier que sur le contexte particulier des migrations internationales de professionnels hautement qualifiés.

\section{BIBLIOGRAPHIE}

Becker H., 1985, Outsiders, Paris : Métaillé.

Berland Y., 2002, Mission « démographie des professions de santé », rapport n ${ }^{\circ}$ 2002135, Paris : ministère de la Santé, de la famille et des personnes handicapées.

Bessiere S., Breuil-Genier P., Darrine S., 2004, La démographie médicale à l'horizon 2025 : une actualisation des projections au niveau national, Études et Résultats, DREES, $\mathrm{n}^{\circ} 353$.

Bliez V., 2006, «La gériatrie recrute » attractivité et fidélisation en gériatrie, Quelle stratégie pour le directeur de soins?, Mémoire de l'école des hautes études en santé publique, Rennes : EHESP.

Chasles V., Denoyel A. et Vincent C., 2013, La démographie médicale en France, le risque des déserts médicaux. L'exemple de la Montagne ardéchoise, Géoconfluences, p.1-13.

CONSEIL NATIONAL DE L'ORDRE DES MEDECINS, Atlas de la démographie en France, Situation au 1 er janvier 2013, Paris : CNOM 
Courgeau D., 1985, Bases théoriques et modèles pour une enquête sur la biographie familiale, professionnelle et migratoire, Espace Populations et Sociétés, $n^{\circ}$ 1, p. 240-247.

Dalkilic S., Meric M. et Trujillo Guiterrez P., 2008, Évaluation au sein de l'Union européenne de la qualité des diplômés internationaux en médecine (DIM) venant de l'extérieur de l'UE - Rapport PADHUE, Paris : Fédération des Praticiens de Santé (FPS).

Descours C., 2003, Propositions en vue d'améliorer la répartition des professionnels de santé sur le territoire, Paris : ministère de la Santé, de la famille et des personnes handicapées.

Drexler A., 2008, Le défi du recrutement des médecins à diplôme étranger dans les hôpitaux publics, Mémoire de l'école des hautes études en santé publique, Rennes : EHESP.

Dormont B. et Samson A.L, 2008, Démographie médicale et carrières des médecins généralistes : les inégalités entre générations, Économie et statistiques, ${ }^{\circ}{ }^{\circ} 414$, p.3-30.

Grimaldi A., 2009, l'hôpital malade de la rentabilité, Paris : Fayard.

Groupe de réflexion sur l'approche biographique (GRAB), 1999, Biographies d'enquêtes : Bilan de 14 collectes biographiques, Paris : PUF/DIFFUSION.

Hartzfeld C., Boidé M. et Baumelou A., Professionnels de santé non citoyens européens et/ou à diplôme non communautaire : leur difficile intégration dans le système français, cas des médecins et des infirmiers, Hommes et migrations, $\mathrm{n}^{\circ}$ 1282, p.90-101.

Lapeyre N. et Le Feuvre N., 2000, Féminisation du corps médical et dynamiques professionnels dans le champ de la santé, Revue française des affaires sociales, $\mathrm{n}^{\circ} 1, \mathrm{p} .59-81$.

Levy D., 2011, Le métier de médecin aujourd'hui, Revue française des affaires sociales, $\mathrm{n}^{\circ} 1, \mathrm{p}$. 299-310.

Lochard Y., Meilland C. et Viprey M., 2007, La situation des médecins à diplôme hors UE sur le marché du travail. Les effets d'une discrimination institutionnelle, Revue de l'Institut de recherches économiques et sociales (IRES), $\mathrm{n}^{\circ}$ 53, p.83-110.

Observatoire national de la démographie des professions de santé, 2004, Les médecins : projections démographiques à l'horizon 2025 par région et spécialité, Tome 2 du rapport annuel 2004, Paris : ONDPS.

Picheral H.et Vigneron E., 1993, La mobilité des médecins en France : du lieu de formation au lieu d'exercice, Espaces, populations, sociétés, Hommage à Daniel Nion, p. 45-54.

Séchet R. et Vasilcu D., 2012, Les migrations de médecins roumains vers la France, entre démographie médicale et quête de meilleures conditions d'exercice, Norois, Environnement, Aménagement et Société, $\mathrm{n}^{\circ}$ 223, p. 63-76 doi:10.4000/norois.4203

Tonneau D., 2003, La réduction du temps de travail dans les hôpitaux publics : des difficultés liées à l'organisation, document de travail, Série Études, n³5, Paris : DREES.

Wallach D., 2011, Numerus clausus , pourquoi la France va manquer de médecins ?, Paris : Folio.

\section{NOTES}

1. Institut de recherche et documentation en économie de la santé.

2. Observatoire national de la démographie des professions de santé. 
3. Cet article se base sur un travail de thèse effectué, au sein du laboratoire MIGRINTER (UMR CNRS 7301) et du département de géographie de l'Université de Poitiers, par Victoire Cottereau sous la direction d'Emmanuel Ma Mung et d'Anne-Cécile Hoyez. Cette recherche doctorale a été financée par la MiRe-DREES (Mission Recherche Direction de la Recherche, des Études, de l'Évaluation et des Statistiques).

4. L'acronyme PADHUE n'est pas une appellation officielle, dans le sens où elle n'apparaît pas dans les textes juridiques français. Les dénominations présentes dans les textes de loi sont le plus souvent : les médecins titulaires d'un diplôme obtenu dans un État non membre de l'Union européenne ou les médecins titulaires d'un diplôme hors Union européenne. Exclues des travaux de recherche en raison de leur longueur excessive, les appellations "officielles » laissent place à différents termes génériques. L'acronyme PADHUE est l'un des plus utilisés, car il indique clairement que c'est le lieu d'obtention du doctorat qui fait qu'un médecin appartient ou non à cette catégorie.

5. Décret n ${ }^{\circ}$ 81-291 du 30 mars 1981 et décret n ${ }^{\circ}$ 87-788 du 28 septembre 1987.

6. Cette frise chronologique synthétique regroupe les diplômes complémentaires de médecine destinés aux PADHUE, les procédures et concours ouverts aux PADHUE pour obtenir l'autorisation d'exercice de la médecine en France ainsi que la législation concernant le recrutement de ces médecins. Les législations sont synthétisées et nous invitons le lecteur à consulter directement les législations pour de plus amples précisions.

7. Loi ${ }^{\circ}$ 98-461 du 13 juin 1998 d'orientation relative à la réduction du temps de travail et loi ${ }^{\circ}{ }^{\circ} 2000-37$ du 19 janvier 2000 relative à la réduction négociée du temps de travail.

8. La réforme de la médecine, initiée par le professeur Robert Debré, se concrétise par l'ordonnance $\mathrm{n}^{\circ}$ 58-1373 du 30 décembre 1958, relative à la création de centres hospitaliers et universitaires ( $\mathrm{CHU}$ ), à la réforme de l'enseignement médical et au développement de la recherche médicale. Elle instaure la fusion de la faculté de médecine et de la clinique au sein de CHU et crée un corps de professeurs hospitaliers et universitaires temps (PU-PH).

9. Il s'agit de tous les praticiens en exercice dans les hôpitaux publics, qu'ils travaillent à temps plein ou à temps partiel.

10. Bien que les pays d'origine de ces médecins fassent aujourd'hui partie de l'UE, ces praticiens sont considérés comme étant des PADHUE, car ils ont été obligés de passer des équivalences pour faire reconnaître leur diplôme.

11. Dans la majorité des cas, le pays d'origine est identique au pays d'obtention du doctorat de médecine.

12. Dans la plupart des pays d'origine, il est impossible d'exercer en effectuant un diplôme de spécialité. Le fait de pouvoir faire une spécialité en France tout en ayant l'autorisation de travailler en parallèle a été une véritable opportunité de devenir spécialiste pour des médecins qui n'avaient pas les moyens de financer leur cursus de spécialité dans leur pays d'origine.

13. Par exemple, les écoles de spécialités ne sont apparues qu'au fil des années 2000 à Madagascar, obligeant les médecins qui souhaitaient devenir spécialistes de partir faire leur spécialité en France.

14. Le cas le plus fréquemment rencontré est celui de médecins diplômés en Algérie venus en France au début des années 1990 qui avaient décidé d'attendre que l'Algérie 
soit pacifiée pour y retourner. Finalement, le conflit ayant duré une dizaine d'années, les médecins ont construit leur vie en France et ils n'ont pas souhaité repartir au moment où l'occasion s'est enfin présentée.

15. Les PADHUE rencontrés qui possédaient le plein exercice de la médecine ont en moyenne mis douze ans pour obtenir la plénitude d'exercice.

16. Union des républiques socialistes soviétiques

\section{RÉSUMÉS}

Depuis les années 1980, l'évolution de la démographie médicale française engendre une inégale distribution des praticiens sur le territoire national. Ces déséquilibres sont en partie liés à des déficits de praticiens dans les hôpitaux de pôles urbains secondaires et à des stratégies d'évitement de certaines zones urbaines défavorisées ou rurales par les nouvelles générations de médecins. Cette problématique a ainsi créé une possibilité pour les praticiens à diplôme hors Union Européenne de venir travailler en France, pour pallier à des déficits dramatiques au sein de structures hospitalières. L'objectif de cette recherche est d'étudier la situation des PADHUE au sein de la région Poitou-Charentes, région touchée par la "pénurie médicale partielle ». Elle s'interroge sur la répartition géographique et les conditions d'exercice de ces médecins ainsi que sur les parcours et les projets migratoires de ces migrants hautement qualifiés qui exercent dans un secteur-clé: celui de l'hôpital.

Since the 1980s, the evolution of the French medical demography has led to an uneven distribution through the national territory. These imbalances are partially due to practitioners' deficits in the hospitals of secondary urban centers and to strategies of avoidance of certain disadvantaged or rural urban areas by the new generations of doctors. This problematic situation has created a possibility for foreign practitioners, qualified outside the European Union to come and work in France in order to alleviate the dramatic deficits within hospitals.

The objective of our research is to study the situation of these particular doctors within the region of Poitou-Charentes, one of the areas affected by the "partial medical shortage". At the crossroads between the academic fields of international migrations and geography of health, this study analyzes the presence and role of these doctors within the French health system, their geographical distribution as well as their particular conditions of exercise. The specific context is examined by looking through the trajectory and migratory projects of these foreign practitioners qualified outside the European Union working in the public hospitals of the region PoitouCharentes. The methodology used in this present research combines quantitative and qualitative data. The qualitative data was provided through the conduct of 80 interviews with doctors and with the administrative personnel (direction / human resources).

The public hospitals of the region Poitou-Charentes has offered a privileged entry for the study of the particular situation of the foreign doctors qualified outside the European Union practicing in France. The conduct of an inventory listing all current practitioners within the public hospitals as of January 1st, 2011 brings new data including all doctors (number, distribution, etc.) and specific to the foreign practitioners qualified outside the European Union. Representing a proportion of about $10 \%$ of all doctors working in the public sector, these highly qualified migrants play a real role of adjustment and balance within a healthcare system that has been 
submitted to national and regional disparities in the distribution of the medical staff.

For the past several years, these doctors have gradually come to enlarge the staff of hospitals that were the most abandoned by medical graduates in France. By doing so, they have been compensating the negative impacts on medical demography caused by the numerus clausus and the reforms of the medical schools. Furthermore, the analysis of the assigned departments of these practitioners through the inventory illustrates how they have been filling the least attractive medical specialties and the most in need of personnel, thus highlighting the original and essential role they play in the functioning of the French healthcare system.

The study of the countries where doctors obtained their medical qualifications reveals that these particular practitioners are mainly qualified in old French colonies and protectorates, making France a privileged destination due to the language, the strong historic links and because of the reputation of the French healthcare system. The analysis of the migratory projects of these doctors has showed that economic motivations are not a fundamental factor in this migration. The majority of these doctors are initially motivated by the offer of diplomas in specific specializations proposed by France to foreign practitioners. Other subsequent factors and events (personal, family-related, etc.) often lead to the abandonment of the initial project, which usually includes an eventual return to the country of origin. The original student mobility is gradually transformed into a long-term international migration. The two main elements usually refraining from the initial project of return are the entitlement to practice and the French nationality, since their acquisition is a much complicated process. Many doctors have recognized that these elements, combined with a greater job security and higher income, have put a definite stop in their project to return.

These doctors are however subjected to a specific and unstable legislation generating numerous obstacles in their professional paths, which makes it difficult for them to fully incorporate the French medical community. Through their discourses and trajectory, we come to reveal the surprising complexity of their careers and their strategies of adaptation that we have coined under the notion of itinerant career allowing us to understand the steps undertaken by these doctors to fully integrate the corporate association. Whether they become mobile or temporarily change professions, these doctors demonstrate a constant capacity of adaptation that is forged by opportunities and strategies.

Although often seen and referred to as "the invisible people of the hospital", these doctors have been essential in the French healthcare system for almost half a century.

\section{INDEX}

Mots-clés : praticiens, métier, migrants, médecins, carrière

Index géographique : France, Europe, Poitou-Charentes

\section{AUTEUR \\ VICTOIRE COTTEREAU}

Doctorante, Laboratoire MIGRINTER (UMR CNRS 7301), Poitiers 